\title{
ENVIRONMENTAL SUSTAINABILITY AND QUALITY OF LIFE: IMPLICATIONS FOR PSYCHOLOGICAL INTERVENTIONS
}

\author{
IRINA G. MALKINA-PYKH ${ }^{1} \&$ YURI A. PYKH ${ }^{2}$ \\ ${ }^{1}$ St. Petersburg State Institute of Psychology and Social Work, Russia \\ ${ }^{2}$ St. Petersburg Mathematical Society, Russia
}

\begin{abstract}
The results of the previous stage of our study of the associations between quality of life (defined as subjective well-being (SWB)) and environmental sustainability revealed that: 1) objective well-being may be compatible with environmental sustainability, often due to synergies arising in terms of reduced pollution and health benefits; 2) well-being and environmental sustainability may be incompatible with one another if well-being is defined as the satisfaction of preferences, psychological well-being, and/or SWB; 3) people with significantly different levels of consumption satisfaction also had significantly different levels of SWB as well as of all other personality variables under consideration. Thus, we hypothesize that it is possible that unhappiness motivates people to focus on material wealth. People who strive for money and goods might suffer from underlying feeling of insecurity. Recent research suggests that happiness can, to some degree, be changed. The first aim of the curren study is to present the psychological intervention (rhythmic-movement therapy) for increasing SWB in general and consumption satisfaction levels in particular. The second aim of our study was to propose generalized multiplicative model (GMultM) for assessing outcome of rhythmic movement therapy. The study was conducted in the framework of Mental Health Management Program organised in the "Human Ecology" department of the Research Center for Interdisciplinary Environmental Cooperation of Russian Academy of Sciences, St. Petersburg, Russia. The results of our study revealed that psychological intervention can be regarded as powerful tool to help participants to shift to more intrinsic goals, reduce materialistic tendencies and thus potentially increase pro-environmental behaviour.

Keywords: quality of life, subjective well-being (SWB), environmental sustainability, personality variables, psychological interventions, generalized multiplicative model (GMultM).
\end{abstract}

\section{INTRODUCTION}

Since 1987, the term "sustainable development" has been used to denote economic, social, and environmental dimensions of our future survival [1]. Environmental sustainability means that all countries need to secure sufficient quality - in the short and the long term - of natural resources, ecosystems, and the diversity of plant and animal species, including the human living environment. Oskamp [2] pleaded for social science to address sustainable development, referring to the "threat of population growth", the "trap of overconsumption", and the "tragedy of underconservation".

After 30 years of environmental policy making some progress in the field of environmental protection has been achieved. In many domains, however, the steady growth in human population, consumption, and technological power is overtaking the environmental improvements achieved [3].

Thus, following a classic formula [4], total environmental resource use (involving wasteful emissions) is a multiplicative function of population, consumption and technology. The basic formula reads: $I=P * A * T$ where $I$ stands for environmental impact, $P$ for population, $A$ for average affluence (consumption per person), and $T$ for the average resource intensity of the technology used per unit of production. It recognises that as human populations continue to grow, material consumption intensifies and production technology 
further expands, the quantity and quality of environmental resources keep steadily decreasing. To reduce environmental damage and risks significant changes are needed in consumer behaviour as well as in manufacturers' products and supply of goods and services, and in people's ability to fulfil their needs and values in a gratifying and sustainable way, using broader-than-material notions of quality of life [5]. However, Sanne [6] argues that most consumers are "locked-in" by social structures and processes that strongly induce environment-burdening patterns of consumption. Such reasoning underscores the importance of psychological changes to achieve environmental sustainability.

Consumerism (materialism) has been conceptualized as a value structure that emphasizes the importance of material possessions and the pursuit of personal wealth [7]. Although consumer values have often been examined at a cultural level [8], psychologists have also studied how they operate on an individual level (e.g. [9]). People possessing high levels of this value tend to be more focused on the material needs of the self and pay less attention to communal goals [10]. Associated with this lack of other-focus, materialist individuals also demonstrate lower levels of empathy, lack of gratitude, and greater levels of relationship conflict (e.g. [11]).

Changing consumer behaviours - and in particular motivating more sustainable behaviours - is far from straightforward. The evidence suggests that policies that act upon objective circumstances may not have the desired effect unless attitudes, perceptions, expectation and attitudes are considered. Some studies explored the effectiveness of incentives and disincentives in promoting pro-environmental behaviours and established that such behaviour can be motivated by the manipulation of material reward, whether token or real [12]. However, it was two undesirable properties of this approach that encouraged researchers to pursue other motivations. It turned out that incentives needed constant reintroduction to remain effective and they proved to be less reliable than we had hoped [13].

Attempts to understand the psychological factors contributing to consumerism have largely focused on the role of values, beliefs, and personal norms (e.g. [14]). According to these models, specific environmental behaviours are motivated when a personal norm associated with a particular value becomes activated. Values themselves are thus understood as higher-order life orientations that inform one's daily goal-directed behaviours [15].

Just as researchers have described the structure of human values, parallel developments in trait theory have produced the "Big Five" model of personality and its association with consumerism (materialism). These five trait domains appear to be cross-culturally valid, are relatively stable across the lifespan, and can be used to predict a variety of real-world outcomes [7].

Another distinct characteristic of materialistic values is that they appear to be negatively related to subjective well-being [16], [17]. Several reports identified consumption at the crux of the relationship between environmental sustainability and well-being. These reports explain that much of the conflict between environmental sustainability and well-being arises when people (driven by extrinsic needs of materialism) try to improve their own well-being through unsustainable consumption patterns which compromises the well-being for future generations through environmental degradation [18].

The predominant assumption of the research on materialism is that purchase and consumption are main motives for placing high importance on money. But there could be other motives for valuing materialistic goals. It's not the money, it's the motives, conclude Srivastava and colleagues [19]. If the motives are social comparison, seeking power, showing off, and overcoming self-doubt, then attaching importance to money is detrimental for wellbeing. Different theoretical perspectives suggest that people who strive for money and goods might suffer from underlying feeling of insecurity [20]. Their dreams frequently include 
themes of death and falling, problems in self-esteem and conflict relationships [21]. Kasser and his collaborators [22] argue that perhaps materialistic pursuits have been evolutionarily ingrained within humans as a way to feel more secure and safe.

One interpretation of this finding is that materialistic individuals tend to be less satisfied with their current resources, always aspiring toward the accumulation of even greater wealth. It should be noted, however, that materialistic values are negatively related to life satisfaction across number of domains, including satisfaction with friends, family, income, and fun [23]. Some authors suggest that this negative relationship with well-being results from the fundamental value conflicts that arise from having a predominantly self-focused worldview [10].

One more possible explanation is that the causal flow between these two psychological factors is bidirectional: happier people act more sustainably and their behaviour makes them feel more happiness. A further and interesting explanation is that when people break out of the conditioned habits of consumption, competition, and inequity practices this is liberating. Individuals take greater control over their life, understanding that they can make conscious choices that contribute to their own well-being, the well-being of others, and the natural environment. In any case, the important fact is that the connection between acting sustainably and happiness seems to be established and environmental psychologists and educators may take advantage of it in their interventional strategies.

The results of the previous stage of our study [18] of the associations between quality of life (defined as subjective well-being (SWB)) and environmental sustainability revealed that: 1) objective well-being may be compatible with environmental sustainability, often due to synergies arising in terms of reduced pollution and health benefits; 2) well-being and environmental sustainability may be incompatible with one another if well-being is defined as the satisfaction of preferences, psychological well-being, and/or SWB; 3) people with significantly different levels of consumption satisfaction also had significantly different levels of SWB as well as of all other personality variables under consideration. Also, in our previous studies we propose the operationalisation of SWB as a multidimensional variable composed of evaluations about different domains of satisfaction with life in a bottom-up or component-based approach [24]-[26]. An advantage of this approach is that judgments on domain satisfaction that are central to determining happiness reflect both subjective factors of the type emphasized in psychology and objective circumstances stressed by economics. Our SWB framework includes five domains (satisfaction with) - material status, health, work, leisure/recreation and personal competence. The following psychological variables were proposed as predictors of SWB level namely dimensions of self-actualisation (time competence and inner directedness), sociotropy, dimensions of perfectionism (personal standards and concern over mistakes/doubts about actions), general locus of control, body image dissatisfaction, neuroticism, alexithymia [25], [26].

Thus, based on the review of the previous studies in the field and our own results we propose the following line of argumentation. We hypothesize that it is possible that unhappiness (low SWB) motivates people to focus on material wealth. A related explanation may also be applied: individuals dispositionally high on broad factors such as neuroticism, perfectionism, or low self-esteem, may be more prone to view money as a means of selfenhancement. So, we argue that increasing of SWB can lead to the increasing of consumption satisfaction and thus to decreasing of consumerism level.

Recent research suggests that happiness can, to some degree, be changed [27]. Recent findings from neuroscience reveal that the brain remains open to new experiences from the environment during the lifespan. This process was called "brain plasticity" and it involves not only the creation of new synaptic connections among neurons but also the growth of new 
neurons [28]. Neuroplasticity is a prerequisite for any enduring change in behaviour, cognition, and emotion, which is the focus of psychotherapy. In order to produce lasting effects, psychotherapy should focus restructuring neural networks, particularly in the subcortical-limbic system which is responsible for unconscious emotional motivations and dispositions. This finding has stimulated the development of interventions that aim at increasing SWB at an individual and public policy levels.

As psychologists have traditionally operated at an individual level, individual interventions provide tools that make people happier (e.g. [29]). A wide variety of interventions that have been developed to increase well-being are based in part on preexisting cognitive behavioural therapies for depression (e.g. [30]). Other promising approaches to increasing of SWB are well-being therapy (e.g. [31]) and positive psychology interventions [32], [33]. Within the embodiment concept the body is linked directly to thought and subsequently to understanding, and that cognitive processes are intrinsically connected to the body. The genesis of thought is mostly unconscious and abstract concepts are mainly presented in a metaphorical manner [34]. Embodiment assumes that cognition evolved for action and because of this, perception and action is linked to each other and to cognition [35], [36].

Another promising body-oriented approach for increasing SWB is dance-movement therapy. In meta-analysis [37] the effectiveness of dance movement therapy (DMT) and the therapeutic use of dance for the treatment of health-related psychological problems were evaluated. Results suggest that DMT and dance are effective for increasing quality of life and decreasing clinical symptoms such as depression and anxiety. Positive effects were also found on the increase of subjective well-being, positive mood, affect, and body image. However, there is not much indication in the subjective well-being literature that body awareness, movement, and body-oriented techniques are used in the increasing subjective well-being. Body-oriented interventions are still in the early stages of development, while interventions that address negative affect, depression, and general unhappiness have a long and deep history. Therefore, while there are some interventions currently in existence, more interventions are needed.

In the present study, we propose the approach of rhythmic movement therapy (RMT) for increasing subjective well-being as well as the level of consumption satisfaction [25], [26]. RMT is one of the creative arts therapies based on the "theoretical interdependence of movement and emotion" [38] along with dance/movement, music, art and drama. RMT as well as many others body-cantered psychotherapies sees the body as part and parcel of the "mental" processes that govern the flexibility and range of our response patterns [39]. Also, in the present study we propose generalised multiplicative model (GMultM) for assessing outcome of rhythmic movement therapy.

\section{METHOD}

The study was conducted in the framework of Mental Health Management Program organised in the "Human Ecology" department of the Research Center for Interdisciplinary Environmental Cooperation of Russian Academy of Sciences, St. Petersburg, Russia.

\subsection{Participants}

At baseline, a total of 273 subjects were recruited among those searching for counselling (psychotherapy) regarding various non-clinical psychological problems: e.g. low self-esteem, family problems, workplace bullying, etc., non-clinical complaints (e.g. diabetes mellitus, rheumatoid arthritis, pulmonary disease, etc.) or mental disorders (e.g. anxiety disorder, 
depression, and panic attacks) were claimed by the participants. We didn't provide the additional screening, but the results of the latest medical examinations were requested from the participants. Demographic items included age and gender, which were assessed with single questions. The participants were aged between 20 and 60 years old, mean age was $37.3 \pm 10.5$ years, $54(20 \%)$ were males and $219(80 \%)$ were females.

The study was approved by the Ethics Committee of I.I. Mechnikov North-West State Medical University, St. Petersburg, Russia, and was performed in accordance with the ethical standards laid down in the 1964 Declaration of Helsinki. All participants signed informed consent form before participating in the study.

\subsection{Measures}

Subjects were assessed with measures listed below. Russian-validated translations of all measures were used.

SWB was measured using Integral Index of Social Well-being (IISW) [40]. The test includes 20 items based on a three-point Likert scale and covers five domains of SWB: work, material well-being, health, leisure/recreation and personal competence. Examples of IISW items include "How satisfied are you with your job?" etc. Six questions reflect the material well-being (current level of consumption satisfaction (LCS)) directly such as "How satisfied are you with your home, apartment or place where you live?", "How satisfied are you with your resources to buy fashionable, beautiful clothing?" and one question we add from the leisure and vacation domain: "How satisfied are you with the length, frequency and quality of vacations you have?" because we argue that it reflects material status indirectly. The responses are tabulated as follows: 1 = "not satisfied"; 2 = "don't know (not of interest)"; 3 = "satisfied". Higher scores show higher level of SWB, maximum score is 60.0. The IISW has demonstrated strong internal consistency and test-retest reliability (0.67). In the experimental sample, the IISW items generated alpha coefficients of 0.87 .

Other measures include Time Competence (Tc) and Inner-Directed (I) scales of Personal Orientation Inventory (POI) [41], General Locus of Control Scale of the Locus of Control Inventory (LOC) [42], Neuroticism (N) Scale of the Eysenck Personality Inventory (EPI) [43]), Toronto Alexithymia Scale-26 (TAS-26) [44], Body Image Test [45], Personal Perfectionism Scale (PPS) of the Multidimensional Perfectionism Scale [46], Sociotropy Scale of Personal Style Inventory (PSI) [47]. Participants were given approximately 1 hour to complete the scales described above.

After baseline assessment all subjects of experimental group were divided into two more groups in accordance with their SWB level: group 1 - (20-40 IISW scores mean very low, low and medium SWB levels) - 156 subjects, 120 females (77\%), 36 males (23\%), mean age $37.6 \pm 11.8$ years $)$ and group $2-(41-60$ scores of IISW mean high and very high SWB levels) - 117 subjects, 99 females (85\%), 18 males (15\%), mean age $36.8 \pm 8.54$ years.

Consumption satisfaction was significantly positively associated with overall subjective well-being $(r=0.741, p<0.01)$.

\subsection{Intervention}

Rhythmic movement therapy (RMT) program was proposed to the subjects of the first group (with very low, low and medium SWB scores on IISW) for increasing their SWB level. All 156 subjects were willing to participate and were randomly assigned ten RMT intervention groups (105 subjects, 24 males, 81 females, mean age $37.6 \pm 11.7$ years) and five waiting list groups (51 subjects, 12 males, 39 females, mean age $37.8 \pm 11.9$ years). Waiting list groups 
didn't receive any counselling or treatment and were contacted after 4 months by psychologists only to administer the assessment scales. The waiting list groups received RMT intervention after completion of post-assessment.

RMT "is a model of psychological intervention that is philosophically and theoretically rooted in body-oriented psychotherapy, dance movement psychotherapy, and rhythmic gymnastics (aerobics)" [26]. The therapeutic work in RMT includes two main components: (1) diagnostic system of core personal problems corresponding with various characters and body types; and (2) rhythmic movement as a medium of change.

Practical applicability of RMT theory and practice is based on the recent advances in developmental psychology and neuroscience such as attachment styles, implicit memory and embodiment theory. Attachment theory provides a useful framework for understanding risk and protective factors in development through each psychosocial crisis described by Erikson in his life cycle model. In more details RMT diagnostic system is described in our previous studies (e.g. [26]).

The RMT intervention was delivered by psychologists who were trained in RMT during a 1-year course. The present RMT intervention consists of 16 once-a-week structured sessions of 45-50 min each in a group setting. Also, within this group setting, each session is highly individualized in accordance with participant's character type, core problems and corresponding results of the initial assessment of psychological characteristics (predictors of SWB level). Each session follows the same basic structure and is devoted to one selected core problem. In the present intervention two sessions were devoted to each core problem. They start with the introduction into the theory of the problem under consideration given by the psychologist (first session on each core problem) and/or with the verbal exchange and feed-back in a circle. The movement part closes with verbal feed-back which lasts about $10-15$ minutes.

All patients of RMT group completed the intervention programs in accord with preplanned schedule of weekly 16 sessions. After this stage was completed, patients of RMT and control groups were repeatedly assessed with the same measures as at the beginning of our study.

\subsection{Generalised multiplicative model of subjective well-being}

Generalised multiplicative model was proposed as the assessment tool for outcome of RMT for increasing SWB and LCS. Previously we studied the performance of "generalised multiplicative models (GMultMs)" which are based on the method of response functions and can be used to identify and characterize nonlinear effect of potential predictors on the SWB level [48]. The main findings of our study [29] indicated that the GMultM not only predicts the level of subjective well-being satisfactorily, but also allows obtaining the partial response functions of psychological predictors of SWB directly as a result of model parameters' estimation.

Based on our operationalisation of SWB the GMultM is looking as follows:

$$
\begin{gathered}
I I S W_{\text {mod }}=I I S W_{\text {norm }} \cdot F_{\text {st }} \\
F_{\text {st }}=f_{1}(I) \cdot f_{2}(T e) \cdot f_{3}(S) \cdot f_{4}(P S) \cdot f_{5}(M D) \cdot f_{6}(L C) \cdot f_{7}(B) \cdot f_{8}(A) \cdot f_{9}(N) \\
f_{j}\left(x_{j}\right)=\alpha_{j} \cdot x_{j}^{\beta_{j}} \cdot \exp \left\{-c_{j}\left\{\frac{x_{j}^{\gamma_{j}}}{x_{j} \max -x_{j}}\right\}\right\} \\
j=1,2,6
\end{gathered}
$$




$$
\begin{gathered}
f_{5}(M D)=\alpha_{5} \cdot M D^{\beta_{5}} \cdot \exp \left\{-c_{5}\left\{\frac{M D}{M D_{\max }-M D}\right\}^{\gamma_{5}}\right\} \\
f_{j}\left(x_{j}\right)=\alpha_{j} \cdot\left(b_{j}-c_{j} \cdot\left(1-\exp \left(-d_{j} \cdot x_{j}^{\gamma_{j}}\right)\right) \quad j=3,4,9\right. \\
f_{j}\left(x_{j}\right)=\alpha_{j} \cdot\left(b_{j}-c_{j} \cdot\left(1-\exp \left(-d_{j} \cdot x_{j}\right)\right)^{\gamma_{j}} \quad j=7,8,\right.
\end{gathered}
$$

where $I I S W_{\text {mod }}, I I S W_{\text {norm }}$ are the modelled actual values of SWB and average normal value obtained during the estimation of model's parameters respectively, $I$ are the scores of inner-directed scale, $T e$ are the scores of time competence scale, $S$ are the scores of sociotropy scale, $P S$ are the scores of personal standards scale, $M D$ are the scores of concern over mistakes, doubts about actions scale, $L C$ are the scores of general locus of control scale, $B$ are the scores of body image dissatisfaction scale, $A$ are the scores of alexithymia scale, $N$ are the scores of neuroticism scale, $\underset{s t}{F} f,{ }_{j}$ are the generalized and partial response functions respectively, $\alpha_{j}, b_{j}, c_{j}, d_{j}, \gamma_{j}, x_{\text {jmax }}$ are parameters for evaluation, $j=1, . ., 9$.

GMultM parameters were identified using the data collected during the first assessment. As function IISW $W_{\bmod }$ is nonlinear in parameters, the problem of parameter estimation of the model (1) can be solved only by numerical methods. The parameters were determined by minimizing the sum of squared differences between estimated data and survey measurements. The corresponding parameters estimation was provided with the module "Isqnonlin" from program package MATLAB Optimization Toolbox. The detailed description of the identification of parameters, validation of the model and computer experiments are presented in [48]. The goodness of model fit was calculated with the mean absolute percentage error (MAPE), also known as mean absolute percentage deviation (MAPD). It expresses accuracy as a percentage, and is defined by the formula:

$$
M A P E=\frac{100}{p} \cdot \sum_{i=1}^{p}\left|\frac{y_{i}-\hat{y}_{i}}{y_{i}}\right|,
$$

where $y_{i}$ is the observed value, $\hat{y}_{i}$ is the predicted value, $p$ is the total number of observed values. MAPE is equal $6.6 \%$ (range from $0.35 \%$ to $17.3 \%$ ) between GMultM and experimental data. While the standard error of SWB measurements in the given sample is equal to $6.9 \%$, the goodness of GMultM fitting seems satisfactory. We argue that MAPE is the appropriate measure of the goodness-of-fit of GMultM because it gives us not only the mean error, but also the errors for each individualized data set within our model. Table 1 contains the values of GMultM parameters.

The parameters of GMultMs, constructed using the response function method, usually have not any meaningful interpretation. However, some combinations of parameters, determining cardinal points of partial response functions (i.e. minimum, optimum and maximum) have specific interpretation and should assume determined values. Such combinations of parameters that determine the important characteristics of the processes 
Table 1: Values of GMultM model parameters.

\begin{tabular}{|c|c|c|c|}
\hline Parameter & Value & Parameter & Value \\
\hline$\alpha_{1}$ & 0.20 & $C_{5}$ & 0.06 \\
\hline$b_{1}$ & 0.86 & $\gamma_{5}$ & 2.87 \\
\hline$C_{1}$ & 0.18 & $M D_{\max }$ & 15.3 \\
\hline$\gamma_{1}$ & 0.79 & $\alpha_{6}$ & 0.63 \\
\hline$x_{1 \max }$ & 12.6 & $b_{6}$ & 0.33 \\
\hline$\alpha_{2}$ & 0.97 & $C_{6}$ & 0.09 \\
\hline$b_{2}$ & 0.04 & $\gamma_{6}$ & 1.29 \\
\hline$c_{2}$ & 0.05 & $x_{6 \max }$ & 12.8 \\
\hline$\gamma_{2}$ & 0.86 & $\alpha_{7}$ & 4.05 \\
\hline$x_{2 \max }$ & 12.6 & $b_{7}$ & 0.25 \\
\hline$\alpha_{3}$ & 0.33 & $c_{7}$ & 1.66 \\
\hline$b_{3}$ & 2.99 & $d_{7}$ & 0.13 \\
\hline$c_{3}$ & 0.73 & $\gamma_{7}$ & 7.59 \\
\hline$d_{3}$ & 0.09 & $\alpha_{8}$ & 0.83 \\
\hline$\gamma_{3}$ & 3.21 & $b_{8}$ & 1.20 \\
\hline$\alpha_{4}$ & 0.44 & $c_{8}$ & 1.80 \\
\hline$b_{4}$ & 2.26 & $d_{8}$ & 0.26 \\
\hline$C_{4}$ & 0.55 & $\gamma_{8}$ & 12.4 \\
\hline$d_{4}$ & 0.28 & $\alpha_{9}$ & 0.92 \\
\hline$\gamma_{4}$ & 1.07 & $b_{9}$ & 0.93 \\
\hline$\alpha_{5}$ & 0.95 & $c_{9}$ & -0.15 \\
\hline$b_{5}$ & 0.03 & $d_{9}$ & 0.0000001 \\
\hline IISWnorm & 18.4 & $\gamma_{9}$ & 7.54 \\
\hline
\end{tabular}

under study and have a specific psychological interpretation we call "connection parameters". Usually we have some knowledge about values of connection parameters such as $x_{i}^{\min }, x_{i}^{\max }, x_{i}^{\text {opt }}$, or can evaluate it from psychological consideration. For example, our finding on locus of control impact on SWB which is presented with unimodal exponential response function $f_{6}(L C)$ is consistent with other results, revealing that internals have significantly better SWB than externals. At the same time the view of partial response function of locus of control supports the idea that optimal values of the construct calculated as: 


$$
x_{o p t}=\frac{a-2^{\gamma-2}(1-\gamma)+2^{\gamma-1}}{3 a+a \gamma-2^{\gamma-1} \gamma},
$$

where ${ }^{a}=x_{\max }^{\gamma-1} \cdot c / b \cdot$ range from 6 to 7 stens. Very high scores of locus of control lead to decreasing of SWB, because subjects could overestimate their control skills and to be disappointed with the results in many life situations.

After the end of RMT program computer experiments with GMultM model and post scores of psychological variables of participants of RMT and control groups were provided with the aim to demonstrate whether intervention-related changes in several psychological variables were mechanisms underlying SWB increasing in subjects participating in RMT program. The mean absolute percentage error of GMultM model simulation of SWB after RMT intervention is equal to $1.3 \%$ (range from $0 \%$ to $23.7 \%$ ) between model output and experimental data. While the goodness of GMultM fit is equal to $6.6 \%$ (i.e. mean absolute percentage error in estimation of model's parameters) the goodness of GMultM pre-post simulations of SWB was accepted as satisfactory. LCS for each participant was calculated using GMulM. Selected results of GMultM pre-post simulations of LCS are presented in Table 2.

Analyzing the data on participants' psychological characteristics before and after RMT program we are able to understand the possible mechanisms underlying SWB/LCS changes during psychotherapy. For example, let us examine the experimental data on subject $\mathrm{N} \mathrm{6,}$ female, 43 years old (see Table 2). We can see that during RMT intervention, time competence and locus of control increased slightly and sociotropy, concern over mistakes and doubts about actions and body image dissatisfaction decreased. As the result of these changes LCS increased from 13 to 18 scores (17 as simulated with GMultM).

Table 2: Pre and post RMT intervention LCS values and scores on the psychological variables used in GMultM simulation experiments.

\begin{tabular}{|c|c|c|c|c|c|c|c|c|c|c|c|c|c|c|c|c|c|c|c|c|c|c|c|}
\hline \multirow[b]{2}{*}{$\mathbf{N}$} & \multirow[b]{2}{*}{$\operatorname{Sex}$} & \multirow[b]{2}{*}{ Age } & \multicolumn{2}{|c|}{ Te } & \multicolumn{2}{|r|}{$I$} & \multicolumn{2}{|c|}{$S$} & \multicolumn{2}{|c|}{$P S$} & \multicolumn{2}{|c|}{$M D$} & \multicolumn{2}{|c|}{$L C$} & \multicolumn{2}{|c|}{ B } & \multicolumn{2}{|c|}{$N$} & \multicolumn{2}{|c|}{$A$} & \multicolumn{3}{|c|}{ LCS } \\
\hline & & & Pre & Post & Pre & Post & Pre & Post & Pre & Post & Pre & Post & Pre & Post & Pre & Post & Pre & Post & Pre & Post & Pre & \begin{tabular}{|l|} 
Post \\
$($ exp $)$ \\
\end{tabular} & $\begin{array}{c}\text { Post } \\
\text { (mod) }\end{array}$ \\
\hline 1 & 1 & 32 & 5 & 8 & 44 & 48 & 96 & 91 & 18 & 18 & 28 & 28 & 2 & 5 & 12 & 12 & 12 & 12 & 77 & 61 & 14 & 18 & 17 \\
\hline 2 & 2 & 36 & 8 & 11 & 30 & 42 & 100 & 89 & 21 & 21 & 54 & 34 & 4 & 6 & 27 & 12 & 9 & 8 & 86 & 65 & 17 & 19 & 18 \\
\hline 3 & 2 & 25 & 7 & 9 & 37 & 42 & 118 & 90 & 22 & 22 & 53 & 34 & 3 & 5 & 36 & 14 & 8 & 8 & 69 & 62 & 11 & 15 & 15 \\
\hline 4 & 2 & 21 & 3 & 7 & 43 & 48 & 86 & 85 & 20 & 20 & 38 & 29 & 6 & 6 & 11 & 11 & 4 & 4 & 75 & 65 & 16 & 17 & 18 \\
\hline 5 & 1 & 65 & 7 & 9 & 44 & 49 & 94 & 89 & 26 & 26 & 40 & 31 & 5 & 6 & 29 & 14 & -4 & -4 & 76 & 68 & 14 & 16 & 17 \\
\hline 6 & 2 & 43 & 6 & 10 & 36 & 40 & 97 & 84 & 13 & 13 & 36 & 31 & 2 & 6 & 26 & 14 & 7 & 6 & 67 & 67 & 13 & 18 & 17 \\
\hline 7 & 2 & 43 & 8 & 10 & 35 & 45 & 108 & 91 & 28 & 21 & 49 & 35 & 0 & 7 & 45 & 23 & 4 & 4 & 73 & 68 & 12 & 17 & 18 \\
\hline 8 & 2 & 30 & 2 & 5 & 29 & 46 & 105 & 92 & 17 & 17 & 46 & 36 & 4 & 7 & 30 & 12 & 10 & 10 & 48 & 48 & 18 & 20 & 21 \\
\hline 9 & 1 & 38 & 8 & 11 & 35 & 47 & 109 & 93 & 21 & 21 & 51 & 36 & 3 & 6 & 21 & 21 & 5 & 5 & 73 & 61 & 18 & 21 & 20 \\
\hline 10 & 2 & 57 & 2 & 4 & 26 & 40 & 117 & 94 & 22 & 22 & 49 & 35 & 4 & 6 & 20 & 20 & 9 & 9 & 94 & 78 & 13 & 16 & 18 \\
\hline
\end{tabular}

Note: Sex: 1 - male, 2 - female; Te - time competence; $I$ - inner directivity; $S$ - sociotropy; PS - personal standards; $M D$ - concern over mistakes, doubts about actions; $L C$ - locus of control; $B$ - body image dissatisfaction; $N$ - neuroticism; $A$ - alexithymia; $L C S(\exp )$ and $L C S($ mod) are experimental and GMultM simulated values of the level of consumption satisfaction. 


\section{DISCUSSION}

Substantial changes in SWB/LCS and underlying psychological state were observed among the participants as a result of RMT intervention. In particular, inner-directedness, time competence and locus of control improved, and sociotropy, perfectionism dimensions, body dissatisfaction, alexithymia and neuroticism decreased. The inclusion of a measurement of such an intrapsychic theoretical construct is of special significance. These findings may support the assumption that solving underlying problems can reduce overt behavioural symptoms, even if the latter are not directly focused on in the session.

The second objective of our study was to demonstrate that GMultM can be successfully used as the individualized assessment tool for evaluating the effectiveness of psychotherapy, because this model is able to predict whether intervention-related changes in several psychological variables are mechanisms underlying SWB change in each individual subject participating in RMT intervention program.

The nonlinear modelling procedure described here is useful for several reasons. First, it provides information about the nonlinear relationships between prognostic factors and subjective well-being that is not revealed by the use of standard statistic and/or linear modelling techniques. Second, GMultM allows predict whether intervention-related changes in several psychological variables are mechanisms underlying subjective well-being change in subjects participating in RMT intervention program. Third, application of the GMultM as assessment tool allows identification of reliable predictors of success in each participant during RMT intervention that could help future programs in several ways. For example, an increased focus could be placed on those intervention components more likely to produce desired outcomes while discarding redundant ones. With a description of critical mechanisms of change, researchers and counsellors may not only improve treatment efficacy and costeffectiveness but also gain insight into aspects that contribute to the persistence of the problem in the first place.

\section{REFERENCES}

[1] Robinson, J., Squaring the circle? Some thoughts on the idea of sustainable development. Ecological Economics, 48, pp. 369-384, 2004.

[2] Oskamp, S., Psychological contributions to achieving an ecologically sustainable future for humanity. Journal of Social Issues, 56, pp. 373-390, 2000.

[3] Vlek, C. \& Steg, L., Human behavior and environmental sustainability: Problems, driving forces, and research topics. Journal of Social Issues, 63(1), pp. 1-19, 2007.

[4] Ehrlich, P.R. \& Holdren, J.P., Impact of population growth. Science, 171, pp. 1212 1217, 1971.

[5] Olander, F. \& Thøgersen, J., Understanding of consumer behaviour as a prerequisite for environmental protection. Journal of Consumer Policy, 18, pp. 345-385, 1995.

[6] Sanne, C., Willing consumers-or locked-in? Policies for a sustainable consumption. Ecological Economics, 42, pp. 273-287, 2002.

[7] Hirsh, J.B. \& Dolderman, D., Personality predictors of consumerism and environmentalism: A preliminary study. Personality and Individual Differences, 43, pp. 1583-1593, 2007.

[8] Campbell, C., The Romantic Ethic and the Spirit of Modern Consumerism, Basil Blackwell: New York, 1987.

[9] Kasser, T. \& Kanner, A.D. (eds), Psychology and Consumer Culture: The Struggle for a Good Life in a Materialistic World, American Psychological Association: Washington, DC, 2004. 
[10] Burroughs, J.E. \& Rindfleisch, A., Materialism and well-being: A conflicting values perspective. Journal of Consumer Research, 29, pp. 348-370, 2002.

[11] Kasser, T., Ryan, R.M., Couchman, C.E. \& Sheldon, K.M., Materialistic values: Their causes and consequences. Psychology and Consumer Culture: The Struggle for a Good Life in a Materialistic World, eds T. Kasser \& A.D. Kanner, American Psychological Association: Washington, DC, pp. 11-28, 2004.

[12] Geller, E.S., Solving environmental problems: A behavior change perspective. Psychology and Social Responsibility: Facing Global Challenges, eds S. Staub \& P. Green, New York University Press: New York, pp. 248-268, 1992.

[13] Katzev, R.D. \& Johnson, T.R., Promoting Energy Conservation: An Analysis of Behavioral Research, Westview Press: Boulder, CO, 1987.

[14] Dietz, T., Fitzgerald, A. \& Shwom, R., Environmental values. Annual Review of Environment and Resources, 30, pp. 335-372, 2005.

[15] Rokeach, M., The Nature of Human Values, Free Press: New York, 1973.

[16] Diener, E. \& Biswas-Diener, R., Will money increase subjective well-being? Social Indicators Research, 57, pp. 119-169, 2004.

[17] Kasser, T. \& Ahuvia, A., Materialistic values and well-being in business students. European Journal of Social Psychology, 32, pp. 137-146, 2001.

[18] Malkina-Pykh, I.G. \& Pykh, Y.A., Environmental sustainability and quality of life: From theory to practice. International Journal of Sustainable Development and Planning, 11(6), pp. 853-863, 2016.

[19] Srivastava, A., Locke, E.A. \& Bartol, K.M., Money and subjective well-being: It's not the money, it's the motives. Journal of Personality and Social Psychology, 80(6), pp. 959-971, 2001.

[20] Kasser, T. \& Sheldon, K.M., On wealth and death: Materialism, mortality salience and consumption behavior. Psychological Science, 11(4), pp. 348-351, 2000.

[21] Kasser, T. \& Kasser, V.G., The dreams of people high and low in materialism. Journal of Economic Psychology, 22, pp. 693-719, 2001.

[22] Kasser, T., Ryan, R.M., Couchman, C.E. \& Sheldon, K.M., Materialistic values: Their causes and consequences. Psychology and Consumer Culture: The Struggle for a Good Life in a Materialistic World, eds T. Kasser and A.D. Kanner, American Psychological Association: Washington, DC, pp. 11-28, 2004.

[23] Richins, M.L. \& Dawson, S., A consumer values orientation scale for materialism and its measurement: Scale development and validation. Journal of Consumer Research, 19, pp. 303-316, 1992.

[24] Malkina-Pykh, I.G. \& Pykh, Y.A., The Method of Response Function in Psychology and Sociology, WIT Press: Southampton and Boston, 2013.

[25] Malkina-Pykh, I.G., Psychological correlates of subjective well-being in the Russian sample. Modern Studies of Russian Society: A Collective Monograph, Unigrafia: Helsinki, pp. 185-199, 2014.

[26] Malkina-Pykh, I.G., Effectiveness of rhythmic movement therapy: Case study of subjective well-being. Body, Movement and Dance in Psychotherapy: An International Journal for Theory, Research and Practice, 10(2), pp. 106-120, 2015. DOI: 10.1080/ 17432979.2014.977822.

[27] Diener, E., Lucas, R.E. \& Scollon, C., Beyond the hedonic treadmill: Revising the adaptation theory of well-being. American Psychologist, 61, pp. 305-314, 2006.

[28] Barbas, H., Anatomic basis of cognitive-emotional interactions in the primate prefrontal cortex. Neuroscience and Biobehavioral Reviews, 19, pp. 499-510, 1995. 
[29] Lyubomirsky, S. \& Layous, K., How do simple positive activities increase well-being? Current Directions in Psychological Science, 22(1), pp. 57-62, 2013.

[30] Diener E. \& Ryan K., Subjective well-being: a general overview. South African Journal of Psychology, 39(4), pp. 391-406, 2009.

[31] Fava, G.A. \& Tomba, E., Increasing psychological well-being and resilience by psychotherapeutic methods. Journal of Personality, 77, pp. 1903-1934, 2009.

[32] Seligman, M.E.P., Rashid, T. \& Parks, A.C., Positive psychotherapy. American Psychologist, 61, pp. 774-788, 2006.

[33] Sin, N.L. \& Lyubomirsky, S., Enhancing well-being and alleviating depressive symptoms with positive psychology interventions: A practice-friendly meta-analysis. Journal of Clinical Psychology, 65, pp. 467-487, 2009.

[34] Lakoff, G. \& Johnson, M., Philosophy in the Flesh: The Embodied Mind and its Challenge to Western Thought, Basic Books: New York, 1999.

[35] Koch, S. \& Fuchs, T., Embodied arts therapies. The Arts in Psychotherapy, 38(4), pp. 276-280, 2011.

[36] Meeks, L., Understanding the personal meaning of the metaphor "Body as Home" for application in Dance/Movement Therapy. MA thesis, Drexel University, 2012.

[37] Koch, S., Kunz, T., Lykou, S. \& Cruz, R., Effects of dance movement therapy and dance on health-related psychological outcomes: A meta-analysis. Arts in Psychotherapy, 41, pp. 46-64, 2014.

[38] Padrao, M.J. \& Coimbra J.L., The anorectic dance: Towards a new understanding of inner-experience through psychotherapeutic movement. American Journal of Dance Therapy, 33, pp. 131-147.

[39] Mowrer, J., Accessing implicit material through body sensations: The body tension sequence. Hakomi Forum, 19-20-21, pp. 145-153, 2008.

[40] Panina, N. \& Golovakha, E., Tendencies in the Development of Ukrainian Society (1994-1998), Sociological Indicators, Institute of Sociology: Kyiv, 2001.

[41] Shostrom, E., Personal Orientation Inventory: Manual, Edits: San Diego, 1974.

[42] Rean, A.A., Handbook of Personality Assessment, St. Petersburg University Press: St. Petersburg, 2001.

[43] Eysenck, H.J. \& Eysenck, S.B.G., Manual of the Eysenck Personality Inventory, University of London Press: London, 1963.

[44] Taylor, G.J., Ryan, D.P. \& Bagby, R.M., Toward the development of a new self-report alexithymia scale. Psychotherapy and Psychosomatics, 44, pp. 191-199, 1985.

[45] Jade, D., Body image test. Therapy of Eating Behaviour, ed. I.G. Malkina-Pykh, EKSMO: Moscow, pp. 347-349, 2002.

[46] Frost, R.O., Marten, P., Lahart, C. \& Rosenblate, R., The dimensions of perfectionism. Cognitive Therapy and Research, 14, pp. 449-468, 1990.

[47] Robins, C.J., Ladd, J., Welkowitz, J., Blaney, P.H., Diaz, R. \& Kutcher, G., The Personal Style Inventory: Preliminary validation studies of new measures of sociotropy and autonomy. Journal of Psychopathology and Behavior Assessment, 16, pp. 277300, 1994.

[48] Malkina-Pykh. I.G., Generalized multiplicative model for assessing outcomes in psychotherapy: Subjective well-being. Nonlinear Dynamics, Psychology, and Life Sciences, 22(2), pp. 191-224, 2018. 